\title{
Abiotrophia defectiva: A Rare Gut Pathogen Resulting Endocarditis in Inflammatory Bowel Disease
}

\author{
Yu Jun Wong ${ }^{1}$, Bee Xian Tan², Maciej Piotr Chlebicki ${ }^{3}$, Brian John Schwender ${ }^{1}$ \\ ${ }^{1}$ Department of Gastroenterology and Hepatology, Singapore General Hospital, Singapore \\ ${ }^{2}$ Department of Microbiology, Singapore General Hospital, Singapore \\ ${ }^{3}$ Department of Infectious Disease, Singapore General Hospital, Singapore
}

\begin{abstract}
Abiotrophia defectiva (formerly referred to as nutritionally variant Streptococcus) is a normal flora of the human gastrointestinal tract. Abiotrophia defectiva is associated with high morbidity and mortality, yet timely diagnosis is challenging. Higher incidence of endocarditis was reported among patients with inflammatory bowel disease (IBD) from a retrospective study. We highlighted the diagnostic and management challenges of a case of rare subacute endocarditis by Abiotrophia defectiva in an IBD patient without predisposing valvular defect, prior central catheter insertion or intravenous drug abuse, suggesting gut translocation as a possible etiology of infective endocarditis among IBD patients. J Microbiol Infect Dis 2017; 7(4):217-219
\end{abstract}

Keywords: Endocarditis, inflammatory bowel disease, ulcerative colitis

\section{INTRODUCTION}

Abiotrophia defectiva was formerly known as a nutritionally variant streptococcus. It is a normal resident in human gastrointestinal and genitourinary tract [1] Abiotrophia defectiva has been reported as a rare cause of subacute and indolent course of endocarditis [2]. It accounts for only $4.3 \%$ to $6 \%$ of all streptococcal endocarditis, mostly among patients with preexisting valvular heart disease [3,4]. Although retrospective studies suggested higher incidence of endocarditis among patients with inflammatory bowel disease (IBD), there were only less than 30 cases reported so far $[5,6]$. We described the diagnostic challenge of Abiotrophia defectiva endocarditis in an IBD patient without predisposing valvular defect, prior central catheter insertion or intravenous drug abuse.

\section{CASE}

A 44-year-old Indian man was diagnosed with Ulcerative Colitis (UC) since 2014. His disease was maintained in clinical and biochemical remission with oral Mesalazine $2 \mathrm{~g}$ twice a day. Surveillance colonoscopy with chromo- endoscopy performed 5 months prior to admission confirmed he is in deep histological remission. One month prior to admission, he was found to have elevated C-reactive protein (CRP) of $39.8 \mathrm{mg} / \mathrm{L}$ from baseline of $1.8 \mathrm{mg} / \mathrm{L}$. There was no clinical evidence of infection or flare of inflammatory bowel disease (IBD) at that time. Computed tomography of abdomen and pelvis performed did not show any intraabdominal collection, abscess or presence of colitis, but incidentally picked up a subcentimetre wedge-shaped area of hypoattenuation at lower pole of left kidney. $\mathrm{He}$ had no history of ischemic or valvular heart disease. In absence of any chest discomfort, urinary or gastrointestinal symptoms or febrile episodes, he was managed conservatively.

One month later, he presented with intermittent rectal bleeding with non-specific abdominal discomfort. Laboratory result showed rising CRP of $87.3 \mathrm{mg} / \mathrm{L}$ and white cell count $\left(16 \times 10^{9} / \mathrm{L}\right)$. $\mathrm{He}$ was admitted and treated initially as mild flare of UC with intravenous hydrocortisone and empirical Ceftriaxone. Initial blood and stool cultures, as well as Clostridium difficile PCR were all negative. Stool calprotectin was 73

Correspondence: Dr. Yu Jun Wong, Academia 20, College Road, Department of Gastroenterology and Hepatology, Singapore 
$\mu \mathrm{g} / \mathrm{g}$. Although his abdominal symptoms resolved shortly after admission, a new ejection systolic murmur was detected. He was afebrile and there were no clinical stigmata of infective endocarditis. A transesophageal echocardiography was performed, which revealed multiple vegetations (ranging from $0.6 \mathrm{~cm}$ to $0.8 \mathrm{~cm}$ ) on mitral valve. Repeated blood cultures subsequently grew Abiotrophia defectiva sensitive to Penicillin ( $\mathrm{MIC}=0.12 \mathrm{mg} / \mathrm{L}$ ) and Vancomycin $(\mathrm{MIC}=0.5 \mathrm{mg} / \mathrm{L})$ after 53 hours of incubation. Identification was done via matrix assisted laser desorption ionization-time of flight (MALDI-TOF) and VITEK 2 GP card (BioMérieux). Dental review excluded oral pathology as a source of Abiotrophia defectiva. $\mathrm{He}$ completed 4 weeks of intravenous Benzylpenicillin and Gentamicin. Although repeated transthoracic echocardiography after treatment showed persistence of vegetations, patient was not keen for surgical intervention as he had no symptoms of heart failure. To date, patient remained well without further embolic or cardiac complications.

\section{DISCUSSION}

Higher incidence of infective endocarditis (IE) was reported among patients with IBD. A German retrospective study showed a 44-fold over-representation of IBD among 213 patients with infective endocarditis [5). Postulations of such findings include: Firstly, intestinal mucosa damage in IBD increases transmural permeability and hence facilitates bacteremia from gut translocation. Secondly, active IBD is often treated with immunosuppressant or immunomodulator therapies that suppress a patient's innate and adaptive immune systems. Lastly, central venous catheters inserted for parenteral nutrition or antibiotic administration may predispose IBD patients to Staphylococcus bacteremia and fungemia [6].

Common organisms that cause endocarditis among IBD patients were Streptococcus spp. and Enterococcus spp. [7,8]. Abiotrophia defectiva is a rare cause of infective endocarditis, accounting for only $4.3 \%$ to $6 \%$ of all streptococcal endocarditis [3]. To our knowledge, this is the first Abiotrophia defectiva endocarditis reported among in an IBD patient without valvular heart disease, suggesting that gut translocation may potentially lead to endocarditis in our patient.

Abiotrophia defectiva was formerly known as a nutritionally variant streptococcus. It is a normal resident in human gastrointestinal and genitourinary tract [1], and usually follows a subacute and indolent course of endocarditis. The challenge of diagnosing Abiotrophia defectiva lies in its fastidious nature. It does not grow on blood agar unless chocolate agar or blood agar supplemented with pyridoxal is used. Of note, Abiotrophia defectiva endocarditis has high mortality compared to endocarditis caused by viridans group streptococci, hence making early diagnosis crucial [9].

\section{Pathogenicity}

The pathogenicity of Abiotrophia defectiva lies in its higher affinity for endocardium because of its ability to secrete exopolysaccharide, enabling it to adhere to fibronectin in the extracellular matrix of heart valve. The same exopolysaccharide also protect them from opsonisation and phagocytosis from hosts' immune system [10]. Under suboptimal nutritional condition Abiotrophia defectiva can exist in cell-wall deficient L-form which renders antibiotic targeting bacteria cell wall such as penicillin ineffective [11].

Antibiotic treatment - resistance and challenges:

The challenge in treating Abiotrophia defectiva lies in its high antibiotic resistance rate and discordance between in vitro susceptibility and clinical outcome. High prevalence of beta-lactam [12] and macrolide [13] resistance among isolates of Abiotrophia defectiva had been reported in small case series. While monotherapy using beta-lactam and macrolide is ineffective, interestingly, combination of penicillin and aminoglycoside significantly enhances bactericidal activity of Abiotrophia defectiva in rabbit. Vancomycin monotherapy was also found to be an effective alternative [14]. On top of that, discordant between in-vitro antibiotic susceptibility and clinical response has been reported. An observational study found that $41 \%$ failed to respond despite being treated with antibiotics that were effective based on in-vitro culture. Early recognition is crucial as the reported relapse rate was up to $17 \%$ despite appropriate antibiotic treatment [15]. Current 
European Society of Cardiology (ESC) guidelines recommend treatment with Penicillin G, Ceftriaxone or Vancomycin for 6 weeks in combination with an aminoglycoside for at least the first 2 weeks for Abiotrophia defectiva endocarditis [16].

Raised inflammatory markers among IBD patients should prompt further work up. Infection should be ruled out especially when patients are in remission for IBD. In this case, the segmental renal infarct showed on computed tomography of abdomen is likely a sequela of cardioembolic event. The auscultated heart murmur prompted us to proceed with transoesophageal echocardiography-which confirmed the diagnosis of endocarditis in this patient. Having a high index of suspicion is crucial to avoid a delay in diagnosis which could result in detrimental complications.

In summary, we reported a rare cause of endocarditis by Abiotrophia defectiva via gut translocation. This case highlighted the importance of prompt investigation in the event of raised inflammatory markers to exclude other causes including infection.

\section{ACKNOWLEDGMENTS}

Conflict of interest: The authors declare no personal or financial conflict of interest.

Funding disclosure: Non to declare

\section{REFERENCES}

1. Bouvet $A$. Human endocarditis due to nutritionally variant streptococci: Streptococcus adjacens and Streptococcus defectivus. Eur Heart J 1995; 16 (suppl B): 24-27.

2. Ramos JN, Santos LSD, Damasco PV, et al. A case report and literature overview: Abiotrophia defectiva aortic valve endocarditis in developing countries. Infection 2014; 42: 579-584

3. Brouqui P, Raoult D. Endocarditis due to rare and fastidious bacteria. Clin Microbiol Rev 2001; 14:177207.

4. Hashimoto $T$, Jodo $S$, Koike $T$, et al. A woman with infective endocarditis caused by Abiotrophia defectiva. Int Med 2004; 43 (10): 1000-1004

5. Kreuzpaintner G, Horstkotte D, Strohmeyer G, et al. Increased risk of bacteria endocarditis in inflammatory bowel disease. Am J Med 1992; 92:391-395.
6. Hatsanos $\mathrm{KH}$, Tsianos EV. The heart in inflammatory bowel disease. Ann Gastroenterol 2002; 15: 124-133.

7. Castillo DJC, Anquita SM, Valles BF, et al. Aneurysm of the anterior mitral valve in a case of aortic endocarditis in a patient with ulcerative colitis. Rev Esp 1998; 51: 604-606.

8. Ali AM, Hamid H, Mohamad AK, et al. Infective endocarditis presented as a right atrium mass in a patient with ulcerative colitis. Case report in cardiology 2015 . Article ID 789170, 3 pages.

9. Tuazon CU, Gill V, Gill F, et al. Streptococcal endocarditis: single vs. combination antibiotic therapy and role of various species. Rev Infect Dis 1986; 8:5460.

10. Frehel C, Hellio R, Bouvet A, et al. Nutritionally variant streptococci develop ultra-structural abnormalities during experimental endocarditis. Microb Pathog 1988; 4:247-255.

11. Rouff KL. Nutritionally Variant Streptococci. Clin Microb Rv 1991: 184-190

12. Touhy MJ, Procop GW, Washington JA, et al. Antimicrobial susceptibility of Abiotrophia adiacens and Abiotrophia defective. Diagn Microbiol Infect Dis 2000; 38:189-191.

13. Liao $\mathrm{CH}$, Teng LJ, Ho SW et al. Nutritionally variant streptococcal infections at a University hospital in Taiwan: disease emergence and high prevalence of beta-lactam and macrolide resistance. Clin Infect Dis 2004; 38:452-455.

14. Bouvet A, Anne CC, C Claude, et al. Comparison of Penicillin and Vancomycin, Individually and in Combination with Gentamicin and Amikacin, in the Treatment of Experimental Endocarditis Induced by Nutritionally variant Streptococci. Antimicrobial Agents and Chemotherapy 1985; 607-611.

15. Stein DS, Nelson KE. Endocarditis due to nutritionally deficient streptococci: Therapeutic dilemma. Rev Infect Dis 1987; 9(5): 908-916

16. Gilbert H, Patrizio L, Manual JA, et al. 2015 ESC guidelines for the management of infective endocarditis. Eur Heart J 2015; 36: 3075-3128. 